THEORY AND METHODS

\title{
Performance of the WHO Rose angina questionnaire in post-menopausal women: Are all of the questions necessary?
}

\author{
D A Lawlor, J Adamson, S Ebrahim
}

See end of article for authors' affiliations

.....................

Correspondence to: Dr D A Lawlor, Department of Social Medicine, University of Bristol,

Canynge Hall, Whiteladies Road, Bristol BS8 2PR, UK; d.a.lawlor@bristol.ac.uk

Accepted for publication 25 November 2002

\begin{abstract}
Objective: To assess the performance of a shortened version of the Rose angina questionnaire focusing on exertional chest pain.

Methods: Cross sectional analysis of 3987 women aged 60 to 79 years from 23 British towns. The performances of definite Rose angina (using data from the full Rose angina questionnaire) and exertional chest pain (using data from a subset of three questions from the Rose angina questionnaire) were assessed against a medical record of angina.

Results: The sensitivity (the proportion with a medical record of angina who were identified as having angina by the questionnaire) was $29.9 \%$ (95\% confidence intervals $25.7 \%$ to $34.4 \%$ ) comparing definite Rose angina to any medical record of angina since 1978 and $50.7 \%$ (45.9\% to 55.5\%) comparing exertional chest pain to any medical record diagnosis of angina. The positive predictive values of both questionnaires were similar. When the two questionnaires were compared with a gold standard of a primary care consultation for angina symptoms within the past five years the sensitivity of definite Rose angina was $33.0 \%(26.9 \%$ to $39.6 \%)$ and that of exertional chest pain was $51.8 \%(45.1 \%$ to $58.5 \%$ ). Although the sensitivity of both versions of the questionnaire was greater in those aged 60-69 years compared with those aged 70-79 years, it remained higher in the exertional chest pain version of the questionnaire than for definite Rose angina based on the full version of the questionnaire in both age groups. Performance of either version of the questionnaire was not affected by occupational social class.

Conclusions: With respect to identifying women with a medical diagnosis of angina or those presenting to primary care with anginal symptoms, these results suggest that a shortened version of the Rose angina questionnaire focusing on exertional chest pain performs better than the full version. Other studies suggest that exertional chest pain is the crucial element of the Rose angina questionnaire with respect to predicting future coronary events. It is concluded that using a shortened version of the Rose angina questionnaire is adequate in epidemiological studies.
\end{abstract}

$\mathrm{T}$ he WHO Rose angina questionnaire ${ }^{1}$ is widely used in epidemiological studies as a standardised method for assessing angina. Although it is a screening tool, rather than a diagnostic test, ${ }^{2}$ and was originally designed for use in men (the figure used to localise the chest pain is a male torso, which is used in study populations of both sexes), it has been found to predict major coronary events in middle aged men ${ }^{3}$ and coronary heart disease mortality in both women and men. ${ }^{4}$ In studies of the ability of the Rose questionnaire to predict future events the crucial predictive component of the questionnaire seems to be experience of exertional chest pain, raising the possibility that some of the questions may be redundant. The entire questionnaire is long, taking up one side of A4 (see fig 1). Shorter questionnaires are one way in which response rates can be increased ${ }^{5}$ and response bias is an important concern in all epidemiological studies.

The aim of this study was to assess the performance of "definite Rose angina" defined using the complete Rose angina questionnaire (fig l) and "exertional chest pain" defined using just three questions from the questionnaire (box l) against a clinical diagnosis of angina in postmenopausal women.

\section{METHODS}

Data from the British Women's Heart and Health Study were used. Full details of the selection of participants and measurements have been previously reported. ${ }^{6}$ Women aged 60-79 years were randomly selected from general practitioner lists in 23 British towns. A total of 4286 women (60\% of those invited) participated and baseline data (self completed questionnaire, research nurse interview, physical examination, and primary care medical record review) were collected between April 1999 and March 2001. Local ethics committee approvals were obtained.

The full Rose Angina questionnaire (fig l) was administered by trained research nurses at the interview. "Definite Rose angina" was defined according to standard criteria as chest pain or discomfort (yes to question 1) that fulfilled all of the following criteria: (a) was brought on by exertion (yes to either question 3 or 4); (b) was situated in the central or left anterior chest (site 4,5 , or 8 on diagram in question 2); (c) forced the subject to slow down or stop (question 5); (d) was relieved if the subject did so (yes to question 6), and (e) was relieved within 10 minutes (question 7). This definition was further subcategorised into severe (grade II) if the exertional chest pain comes on when walking on level ground (yes to question 3) and not severe (grade I) if the exertional chest pain only comes on when hurrying or walking up hill (no to question 3 and yes to question 4). Angina based just on the subset of three questions from the questionnaire (box 1) was defined as exertional chest pain (yes to question 1 and yes to either question 2 or 3). Again this was subcategorised as grade I and grade II as described above.

The performance of these two definitions of questionnaire angina were compared with a clinical diagnosis of angina 
1 Do you ever have any pain or discomfort in your chest? Yes/No

2 Where do you get this pain or discomfort? Please mark $\mathbf{X}$ on the appropriate places

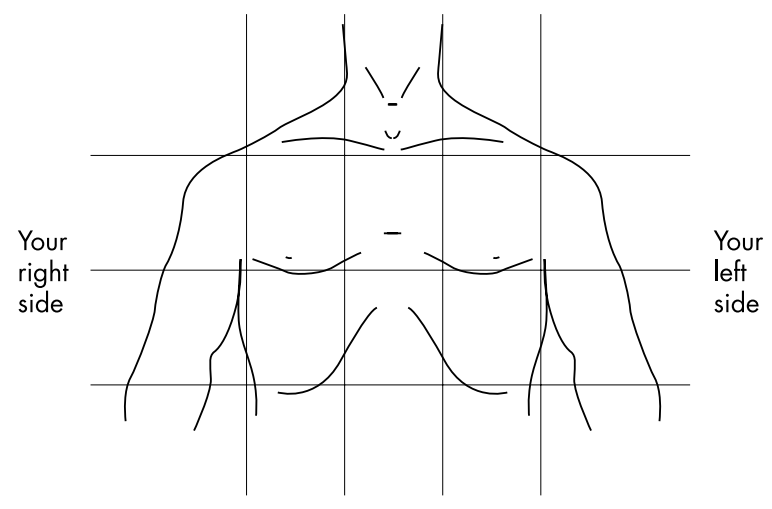

3 When you walk at an ordinary pace on the level does this produce the pain?

Yes/No/Unable

4 When you walk uphill or hurry does this produce the pain?

Yes/No/Unable

5 When you get any pain or discomfort in your chest on walking, what do you do?

Stop Slow down Continue at same pace Not applicable

6 Does the pain or discomfort in your chest go away if you stand still?

Yes/No

7 How long does it take to go away?

10 minutes or less more than 10 minutes

Figure 1 Complete WHO Rose Angina questionnaire.

from the medical records. Detailed reviews of participants' general practice medical records (including general practitioner notes, hospital letters, and computer data) were undertaken independently by a member of the primary care medical team to identify all diagnoses of angina occurring since 1978, with their date of occurrence. The people undertaking the medical record reviews at each practice were unaware of any of the participants responses to the Rose angina questionnaire. Each questionnaire diagnosis of angina was compared with a "gold standard" of any record of a diagnosis of angina since 1978. As the questionnaire refers to symptomatic angina, and some patients with a diagnosis of angina in their medical records may be well controlled and symptom free, we repeated the analyses with a different "gold standard": consultation with a doctor with anginal symptoms within the five years before the Rose angina assessment was carried out.

\section{Statistical analysis}

Sensitivity (the proportion of those with angina in their medical records identified by the questionnaires); specificity (the proportion of those with no diagnosis of angina in their
Box 1 Shortened WHO Rose Angina questionnaire,

focusing on exertional chest pain

1 Do you ever have any pain or discomfort in your chest? - Yes/No

2 When you walk at an ordinary pace on the level does this produce the pain?

- Yes/No/Unable

3 When you walk uphill or hurry does this produce the pain? - Yes/No/Unable

medical records who were defined by the questionnaires as not having angina); positive predictive value (the proportion identified by the questionnaire as having angina who actually had a medical diagnosis), and negative predictive value (the proportion identified by the questionnaire as not having angina who did not have a medical diagnosis) were expressed as percentages, with $95 \%$ confidence intervals calculated using the exact binomial method. Analyses were repeated stratified by age (two age groups: 60-69 years and 70-79 years) and occupational social class (manual occupational class and nonmanual occupational class based on the participant's husband's longest held occupation for married women and her own longest held occupation for single women) to determine whether accuracy was affected by age or social class.

\section{RESULTS}

Of the 4286 participants in the British Women's Heart and Health Study 3987 (93\%) had complete classifiable data for the Rose angina questionnaire; as the subset of "exertional" chest pain questions was derived from the complete questionnaire the response rate was identical for both applications of the questionnaire. Of the 299 women without complete classifiable data 237 had missing data on at least one question and 62 had unclassifiable responses, for example an indication that they were unable to walk on the level (question 3) and then a response indicating that they stopped or slowed down if the pain came on while walking (question 5). These women did not differ from those with complete data with respect to age (68.8 years versus 68.7 years, $\mathrm{p}=0.95)$, medical record diagnosis of myocardial infarction $(2.03 \%$ versus $2.01 \%$, $\mathrm{p}=0.98)$, angina $(11.00 \%$ versus $12.00 \%, \mathrm{p}=0.44)$, stroke ( $1.73 \%$ versus $2.00 \%, p=0.31)$, diabetes $(4.56 \%$ versus $4.68 \%$, $\mathrm{p}=0.96)$ or cancer $(9.08 \%$ versus $9.70 \%, p=0.72)$. All participants had medical record review data. All further presented analyses refer just to the 3987 women with complete data on the angina questionnaire.

Table 1 shows the prevalence of definite Rose angina, exertional chest pain and non-exertional chest pain (as determined by the questionnaire) together with the prevalence of angina determined by the review of medical records and the prevalence of those presenting with anginal symptoms within the past five years. Thirteen per cent of the women had exertional chest pain; $7.7 \%$ definite Rose angina, and $11.0 \%$ a clinical diagnosis of angina in their medical records, with 5.6\% having consulted with anginal symptoms in the past five years. Only 68 (1.7\%, $1.3 \%$ to $2.2 \%)$ women had had revascularisation procedures (coronary artery bypass graft or balloon angiography) and 301 (7.5\%, 6.7\% to $8.4 \%)$ women were taking anti-anginal nitrate medication. Exclusion of these women from subsequent analyses did not materially affect our findings, and all presented results include these 369 women.

Table 2 shows the performance of definite Rose angina and exertional chest pain compared with a clinical diagnosis of angina. The sensitivity was higher with the exertional chest pain $(51 \%)$ than when definite Rose angina (30\%) was used. Specificity was slightly higher for definite Rose angina. The positive predictive value of both were similar. Sensitivity of 
Table 1 Response to WHO Rose angina questionnaire. Women aged 60-79 years

\begin{tabular}{lll}
\hline & $\begin{array}{l}\text { Number/total number } \\
\text { of women }\end{array}$ & $\begin{array}{l}\%(95 \% \text { confidence } \\
\text { intervals) }\end{array}$ \\
\hline $\begin{array}{l}\text { Questionnaire } \\
\text { Exertional chest pain (total) }\end{array}$ & $519 / 3987$ & $13.0(12.0$ to 14.1$)$ \\
$\quad \begin{array}{l}\text { Not severe grade I } \\
\quad \text { Severe grade II }\end{array}$ & $378 / 3987$ & $9.5(8.6$ to 10.4$)$ \\
Definite Rose angina (total) & $141 / 3987$ & $3.5(3.0$ to 4.2$)$ \\
$\quad \begin{array}{l}\text { Not severe grade I } \\
\quad \text { Severe grade II }\end{array}$ & $307 / 3987$ & $7.7(6.9$ to 8.6$)$ \\
Medical record & $232 / 3987$ & $5.8(5.1$ to 6.6$)$ \\
Clinically diagnosed angina & $75 / 3987$ & $1.9(1.5$ to 3.4$)$ \\
Consultation with angina symptoms in past five years & $438 / 3987$ & $11.0(10.0$ to 12.0$)$ \\
\hline
\end{tabular}

Table 2 Sensitivity, specificity, positive predictive value, negative predictive value of definite Rose angina, exertional chest pain against medical diagnosis of angina

\begin{tabular}{|c|c|c|}
\hline & Definite Rose angina & Any exertional chest pain \\
\hline \multicolumn{3}{|c|}{ Gold standard=any diagnosis of angina since 1978 in medical records } \\
\hline Sensitivity \% (95\% CI) & 29.9 (25.7 to 34.4$)$ & 50.7 (45.9 to 55.5$)$ \\
\hline Specificity \% (95\% Cl) & $95.0(94.3$ to 95.7$)$ & $91.6(90.7$ to 92.5$)$ \\
\hline Positive predictive value $\%(95 \% \mathrm{CI})$ & 42.7 (37.1 to 48.4$)$ & 42.8 (38.5 to 47.2$)$ \\
\hline Negative predictive value $\%(95 \% \mathrm{Cl})$ & $91.7(90.7$ to 92.5$)$ & 93.8 (92.9 to 94.6$)$ \\
\hline \multicolumn{3}{|c|}{$\begin{array}{l}\text { Gold standard=medical record of anginal symptoms within the five years before completion of } \\
\text { Rose angina questionnaire }\end{array}$} \\
\hline Sensitivity $\%(95 \% \mathrm{CI})$ & $33.0(26.9$ to 39.6$)$ & $51.8(45.1$ to 58.5$)$ \\
\hline Specificity \% (95\% CI) & $93.8(93.0$ to 94.6$)$ & $89.4(88.4$ to 90.4$)$ \\
\hline Positive predictive value $\%(95 \% \mathrm{CI})$ & 24.1 (19.4 to 29.3$)$ & 22.5 (19.0 to 26.4 ) \\
\hline Negative predictive value $\%(95 \% \mathrm{Cl})$ & $95.9(95.2$ to 96.5$)$ & $96.9(96.3$ to 97.4$)$ \\
\hline
\end{tabular}

Table 3 Sensitivity, specificity, positive predictive value, negative predictive value of definite Rose angina, exertional chest pain against medical diagnosis of angina stratified by age group

\begin{tabular}{|c|c|c|}
\hline & Definite Rose angina & Any exertional chest pain \\
\hline \multicolumn{3}{|l|}{ Age $60-69$ years $(n=2165)$} \\
\hline Sensitivity \% (95\% CI) & 31.1 (29.1 to 33.0$)$ & $56.5(54.4$ to 58.6$)$ \\
\hline Specificity \% (95\% CI) & 95.1 (94.2 to 96.0$)$ & $91.8(90.7$ to 93.0$)$ \\
\hline Positive predictive value $\%(95 \% \mathrm{Cl})$ & 36.2 (34.2 to 38.2 ) & $38.0(36.0$ to 40.1$)$ \\
\hline Negative predictive value $\%(95 \% \mathrm{CI})$ & 93.9 (92.9 to 94.9$)$ & 96.0 (95.1 to 96.8$)$ \\
\hline \multicolumn{3}{|l|}{ Age $70-79$ years $(n=1822)$} \\
\hline Sensitivity \% (95\% CI) & 29.1 (27.0 to 31.2$)$ & 45.2 (42.9 to 47.5$)$ \\
\hline Specificity \% (95\% CI) & 94.9 (93.9 to 96.0$)$ & 91.4 (90.1 to 92.7$)$ \\
\hline Positive predictive value $\%(95 \% \mathrm{Cl})$ & 49.0 (46.7 to 51.3$)$ & 49.8 (47.5 to 52.1$)$ \\
\hline Negative predictive value $\%(95 \% \mathrm{CI})$ & 88.9 (87.5 to 90.3 ) & 90.9 (89.6 to 92.2$)$ \\
\hline
\end{tabular}

both definite Rose angina and exertional chest pain increased when the comparison was made with those who had consulted with symptoms in the past five years. However, exertional chest pain still performed better with respect to sensitivity. The positive predictive values decreased for both tests because the prevalence of women who had presented with symptoms during the previous five years was lower than that for any diagnosis of angina.

Tables 3 shows the analyses stratified by age group (60-69 years versus $70-79$ years). With respect to sensitivity both versions of the questionnaire performed better in women aged 60-69 years than those aged 70-79 years. However, a similar pattern to that for the whole cohort, between the two versions of the questionnaire, remained, with sensitivity being greater in both age groups for exertional chest pain than for definite angina. As the prevalence of angina is greater in older age groups the positive predictive values of both versions of the questionnaire was greater among older women. However, positive predictive value was similar with both versions of the questionnaire (as in the main cohort) in the age stratified analysis. There was no difference in the performance of either version of the questionnaire when analyses were stratified by social class, for example sensitivity of definite Rose angina among women from manual social classes was 29.72 (27.65 to 31.79 ) compared with 28.86 (26.75 to 30.97 ) for non-manual social classes. Similar results for exertional chest pain were 49.00 (46.73 to 51.26 ) and 48.43 (46.08 to 50.77).

\section{DISCUSSION}

Our results suggest that in post-menopausal women using a shortened version of the Rose angina questionnaire, a subset of just three questions, performs as well as the full version. One difficulty with assessing the performance of any screening tool for angina is the lack of a clear gold standard. ${ }^{7}$ However, the consistency of our findings, using independent medical record diagnoses as the gold standard, with those of 


\section{Key points}

- The entire Rose angina questionnaire is long, taking up at least one side of A4

- Response bias is an important problem in epidemiological studies and one way to reduce this is to maximise the response rates by keeping questionnaires as short as possible

- Prospective studies suggest that the crucial component of the Rose angina questionnaire with respect to predicting future coronary events is exertional chest pain

- In this study a shortened version (three questions) of the Rose angina questionnaire focusing on exertional chest pain performed better than using data from the full questionnaire when both were compared with a medical record of a diagnosis of angina or consultation within the past five years with anginal symptoms

- These findings were not changed when results were stratified by age group or by occupational social class

\section{Policy implications}

- A shortened (three question) version of the Rose angina questionnaire is adequate as a screening tool for angina in epidemiological studies

- With either the full or shortened version of the questionnaire at least $50 \%$ of post-menopausal women with a medical record diagnosis of angina will not be identified by the questionnaire

two prospective studies that suggested that questions about exertional chest pain predict future ischaemic events to the same extent as definite Rose angina, in women and men $^{34}$ support the idea that the shortened version of the questionnaire is valid. Though higher for the shortened version of the questionnaire, the sensitivity was low for both versions- the shortened version would identify only $52 \%$ of women with symptoms defined as angina in their medical records over the preceding five years. The remaining $48 \%$ may have been asymptomatic at the time of the questionnaire. However, the low sensitivity of the Rose angina questionnaire highlights its limitations with respect to assessing the population disease burden of angina.

Similar proportions of severe angina $(27 \%$ of those with definite Rose angina and $25 \%$ of those with exertional chest pain) were defined as having severe (grade II) symptoms. The better performance of the shortened version of the questionnaire is therefore unlikely to be attributable to differences in proportions of severity of angina between the two definitions. This study assesses the performance of the full and shortened version of the questionnaire cross sectionally with respect to a clinical diagnosis. An important aspect of the Rose angina questionnaire is its ability to predict future cardiovascular events. With long term follow up of these women we will further assess the performance of the full and shortened version of the questionnaires to determine whether results of previous studies with respect to the prediction of future events are confirmed in this group of post-menopausal women. ${ }^{3}$
In conclusion we would suggest that a shortened version of the Rose angina questionnaire performs better than the full version with respect to identifying patients with clinical angina in post-menopausal women. Although both versions of the questionnaire perform better in younger compared with older women the difference between the full and a shortened version of the questionnaire remained in both age groups suggesting that the shortened version may be suitable for all age groups. Prospective studies suggest that a shortened version of the questionnaire focusing on exertional chest pain may also be adequate for men, ${ }^{34}$ and our results suggest that socioeconomic position does not importantly affect the performance of either version of the questionnaire when administered by a research nurse.

\section{ACKNOWLEDGEMENTS}

The British Women's Heart and Health Study is co-directed by Professor Shah Ebrahim, Professor Peter Whincup, Dr Goya Wannamethee, and Dr Debbie A Lawlor. We thank Carol Bedford, Alison Emerton, Nicola Frecknall, Karen Jones, Mark Taylor, and Katherine Wornell for collecting and entering data, all of the general practitioners and their staff who have supported data collection and the women who have participated in the study.

\section{Contributors}

All authors developed the study aim and design. DAL undertook the initial analysis and coordinated writing of the paper. All authors contributed to the final version.

\section{Authors' affiliations}

D A Lawlor, J Adamson, S Ebrahim, Department of Social Medicine, University of Bristol, Canynge Hall, Whiteladies Road, Bristol BS8 2PR, UK

Funding: The British Women's Heart and Health Study is funded by the Department of Health. DA Lawlor and J Adamson are funded by a Medical Research Council/Department of Health training fellowship. The views expressed in this publication are those of the authors and not necessarily those of the funding bodies.

Conflicts of interest: none declared.

\section{REFERENCES}

1 Rose G. The diagnosis of ischaemic heart pain and intermittent claudication in field surveys. Bull World Health Organ 1962;27:645-58.

2 Rose G, McCartney P, Reid DD. Self-administration of a questionnaire on chest pain and intermittent claudication. Br J Prev Soc Med 1977;31:42-8.

3 Lampe FC, Whincup PH, Wannamethee SG, et al. Chest pain on questionnaire and prediction of major ischaemic heart disease events in men. Eur Heart J 1998; 19:63-73.

4 Hart CL, Watt GC, Davey Smith G, et al. Pre-existing ischaemic heart disease and ischaemic heart disease mortality in women compared with men. Int J Epidemiol 1997:26:508-15.

5 Ransdell LB. Maximising response rate in questionnaire research. American Journal of Health Behaviour 1996;20:50-6.

6 Lawlor DA, Bedford C, Taylor M, et al. Geographical variation in cardiovascular disease, risk factors and their control in older women: British Women's Heart and Health Study. J Epidemiol Community Health 2003;57:134-40.

7 Hlatky MA, Mark DB, Califf RM, et al. Angina, myocardial ischemia and coronary disease: gold standards, operational definitions and correlations. J Clin Epidemiol 1989;42:381-4. 